\title{
Spin kinetic equations in the probability representation of quantum mechanics
}

\author{
Vladimir N. Chernega ${ }^{1}$, Vladimir I. Man'ko' ${ }^{1,2}$ \\ 1 - Lebedev Physical Institute, Russian Academy of Sciences \\ Leninskii Prospect 53, Moscow 119991, Russia \\ 2 - Moscow Institute of Physics and Technology (State University)/ Institutskii per. 9, \\ Dolgoprudnyi, Moscow Region 141700, Russia \\ Corresponding author e-mail: vchernega @ gmail.com
}

\begin{abstract}
We discuss the possibility to formulate the dynamics of spin states described by the Schrödinger equation for pure states and the von Neumann equation (as well as the GKSL equation) for mixed states in the form of quantum kinetic equations for probability distributions. We review an approach to the spin-state description by means of the probability distributions of dichotomic random variables.
\end{abstract}

Keywords: spin states, kinetic equations, Gorini-Kossakowski-Sudarshan-Lindblad (GKSL) equation, Schrödinger equation.

\section{Introduction}

The quantum kinetic equations based on the Schrödinger equation for the wave function [1] and the von Neumann equation for the density matrix [2, 3] have been used to study different physical systems (in particular, the properties of Fermi liquids have been studied in [4, 5, 6, 7, 8, 9, 10, 11]). The important role of spin states in the dynamics of such systems as electron liquids in metals was considered in the presence of magnetic field in [12].

The aim of this paper is to review recent approach to the description of quantum states by means of probability distributions of standard classical-like random variables [13, 14, 
15, 16, 17, 18. This approach provides the possibility to construct the map of density operators and state vectors belonging to a Hilbert space of quantum-system states onto probability distributions, which means that all equations, including the Schrödinger equation for state vectors and the von Neumann equation for density operators, can be mapped onto kinetic equations for the probability distributions.

We present this approach on the example of a system with discrete variables like the spin-1/2 system and formulate the generic method for deriving the kinetic equations for classical-like probability distributions describing any quantum system. Some aspects of such approach are presented in [19, 20, 21, 22, 23, 24].

This paper is organized as follows.

In Sec. 2, the spin-1/2 states are identified with the probability distribution of dichotomic random variables. In Sec. 3, the quantum evolution of the spin- $1 / 2$ density matrix is presented in the form of a kinetic equation for dichotomic variables describing the spin states. In Sec. 4, generic systems with discrete and continuous variables are considered using the probabilities describing their states. Conclusions and prospects are given in Sec. 5.

\section{Spin-1/2 Density Matrix}

The pure state of the spin- $1 / 2$ system (e.g., the electron spin in a metal) is identified with the state vector $|\psi\rangle$, which has complex components $a$ and $b$ satisfying the normalization condition $|a|^{2}+|b|^{2}=1$. In the case of mixed state of the spin system, the density $2 \times 2$ matrix $\rho$, with complex matrix elements $\rho_{11}, \rho_{12}, \rho_{21}=\rho_{12}^{*}$, and $\rho_{22}$, such that $\rho=\rho^{\dagger}, \operatorname{Tr} \rho=1$, and $\rho \geq 0$, is identified with the state.

The pure state has the density matrix $\rho_{\psi}=|\psi\rangle\langle\psi|$, where the column vector $|\psi\rangle$ is considered as a rectangular matrix with two rows, and the vector $\langle\psi|$ is considered as the matrix with two columns, i.e., $\langle\psi|=(|\psi\rangle)^{\dagger}$. The matrix $\rho_{\psi}$ is defined as a $2 \times 2$ matrix given by the product of two rectangular matrices $\rho_{\psi}=(|\psi\rangle)(|\psi\rangle)^{\dagger}$.

The evolution equation for the spin system with a Hamiltonian $H$, which is the $2 \times 2$ matrix with matrix elements $H_{11}, H_{12}, H_{21}=H_{12}^{*}$, and $H_{22}$, such that $H=H^{\dagger}$, 
has the form of the Schrödinger equation

$$
i \frac{\partial}{\partial t}|\psi(t)\rangle=H(t)|\psi(t)\rangle, \quad \hbar=1
$$

The unitary evolution of the state vector, i.e., $|\psi(t)\rangle=U(t)|\psi(0)\rangle$, where the unitary $2 \times 2$ matrix $U(t)$ has the matrix elements $U_{11}(t), U_{12}(t), U_{21}(t)$, and $U_{22}(t)$, satisfies the equation

$$
i \frac{\partial U(t)}{\partial t}=H(t) U(t), \quad U(0)=1
$$

and one has the unitarity condition $U(t) U^{\dagger}(t)=1$. For the time-independent Hamiltonian, $U(t)=\exp (-i t H)$ and the density matrix $\rho(t)$ of arbitrary states evolves as $\rho(t)=$ $U(t) \rho(0) U^{\dagger}(t)$.

We address now the following problem: how to describe the discussed form of quantum dynamics using standard probability distributions and their time dependence.

Following [13, 25, 26, 27], one can check that, if we denote the matrix elements of the spin density matrix as $\rho_{11}=p_{3}$ and $\rho_{12}=p_{1}-1 / 2-i\left(p_{2}-1 / 2\right)$, the real numbers $0 \leq p_{1}, p_{2}, p_{3} \leq 1$ have the physical meaning of probabilities to have spin projections on perpendicular axes $x, y$, and $z$ equal to $+1 / 2$. One can check this fact by calculating the trace $\operatorname{Tr}\left(\rho \rho_{k}\right)$ which, due to Born's rule, is the probability to have in the state $\rho$ the properties associated with the states $\rho_{k}$. If we choose three density matrices $\rho_{k}, k=1,2,3$ as density matrices

$$
\rho_{1}=\frac{1}{2}\left(\begin{array}{cc}
1 & 1 \\
1 & 1
\end{array}\right), \quad \rho_{2}=\frac{1}{2}\left(\begin{array}{cc}
1 & -i \\
i & 1
\end{array}\right), \quad \rho_{3}=\left(\begin{array}{ll}
1 & 0 \\
0 & 0
\end{array}\right),
$$

these matrices describe the states with spin projection $+1 / 2$ onto the directions $x$, $y$, and $z$, since they correspond to eigenvectors $\left|\psi_{1}\right\rangle,\left|\psi_{2}\right\rangle$, and $\left|\psi_{3}\right\rangle$ of the Pauli matrices $\sigma_{1}, \sigma_{2}$, and $\sigma_{3}$; the obtained numbers $p_{1}, p_{2}$, and $p_{3}$ have exactly the meaning of probabilities. These three numbers can be used to get all the matrix elements of the density matrix $\rho$ expressed as linear combinations of the probabilities.

Thus, we obtain an invertible map $\rho \leftrightarrow p_{1}, p_{2}, p_{3}$ of the density matrix onto three probability distributions $\left(p_{1}, 1-p_{1}\right),\left(p_{2}, 1-p_{2}\right)$, and $\left(p_{3}, 1-p_{3}\right)$ of dichotomic random variables. The probability distributions can be interpreted as probabilities describing statistics of three nonideal classical coins. 
For classical coins that are independent (there is no correlations), the numbers $p_{1}, p_{2}$, and $p_{3}$ satisfy only one condition $0 \leq p_{1}, p_{2}, p_{3} \leq 1$.

For spin states, the nonnegativity of the density matrix provides the constraint $\left(p_{1}-\right.$ $1 / 2)^{2}+\left(p_{2}-1 / 2\right)^{2}+\left(p_{3}-1 / 2\right)^{2} \leq 1 / 4$ that corresponds to the presence of quantum correlations of the spin-projections onto three different directions.

As it was always considered before, the quantum nature of the system behavior (like the spin-1/2 system) needed the formalism of Hilbert space vectors and operators acting in this space to develop the theory of physical phenomena. But it turned out that, as in classical theory, it is sufficient to use the formalism of standard probability theory to describe the states of the systems, e.g., the spin- $1 / 2$ system. The only ingredient introduced by the quantum mechanics formalism is the fact that in nature there exist quantum correlations, which impose extra constraints onto the probability distributions expressed in terms of inequalities, which do not exist in corresponding classical systems like three coins.

The three probability distributions can be considered as conditional probability distributions $P(m \mid j), m= \pm 1 / 2, j=1,2,3$; here, $P(1 / 2 \mid 1)=p_{1}, P(1 / 2 \mid 2)=p_{2}$, and $P(1 / 2 \mid 3)=p_{3}$. The joint probability distribution of two random variables $\mathcal{P}(m, j)$ provides three conditional probability distributions due to Bayes' formula $\mathcal{P}(m, j)=$ $P(m \mid j)\left(\sum_{m=-1 / 2}^{1 / 2} \mathcal{P}(m, j)\right)$. Here, $\sum_{m=-1 / 2}^{1 / 2} \mathcal{P}(m, j)$ is the marginal probability distribution to have a random variable $j$, which plays the role of the coin number in the classical case - the number $P(m \mid j)$ is the probability of the spin projection $m$ on the direction of the axes $x, y$, and $z$ in the experiments where the spin projections $+1 / 2$ onto the three directions are measured.

A simple example of such marginal probability distribution is $\sum_{m=-1 / 2}^{1 / 2} \mathcal{P}(m, j)=$ $(1 / 3,1 / 3,1 / 3)$. In this case, the joint probability distribution $\mathcal{P}(m, j)$ contains six probabilities expressed by the probability 6 -vector $\overrightarrow{\mathcal{P}}=\frac{1}{3}\left(p_{1}, 1-p_{1}, p_{2}, 1-p_{2}, p_{3}, 1-p_{3}\right)$. 


\section{Evolution of the Spin Quantum State as the Time Dependence of the Probability Distribution}

The unitary evolution equation for the density matrix of the spin- $1 / 2$ system, associated with the Hamiltonian $H$, which is a Hermitian $2 \times 2$ matrix with matrix elements $H_{j k}$, $j, k=1,2$, reads

$$
i \frac{\partial \rho}{\partial t}=H \rho-\rho H
$$

it is the kinetic equation for the probabilities $p_{1}(t), p_{2}(t)$, and $p_{3}(t)$ of the form

$$
i\left(\begin{array}{cc}
\dot{p}_{3} & \dot{p}_{1}-i \dot{p}_{2} \\
\dot{p}_{1}+i \dot{p}_{2} & -\dot{p}_{3}
\end{array}\right)=\left[\left(\begin{array}{cc}
H_{11} & H_{12} \\
H_{21} & H_{22}
\end{array}\right),\left(\begin{array}{cc}
p_{3} & p_{1}-1 / 2-i\left(p_{2}-1 / 2\right) \\
p_{1}-1 / 2+i\left(p_{2}-1 / 2\right) & 1-p_{3}
\end{array}\right)\right]
$$

This von Neumann equation for the density matrix is equivalent to a linear differential equation for the probability 6 -vector $\overrightarrow{\mathcal{P}}(t)$ of the form $\frac{d \overrightarrow{\mathcal{P}}}{d t}=\hat{H} \overrightarrow{\mathcal{P}}+\vec{\Gamma}$. The matrix elements of the $6 \times 6$ matrix $\tilde{H}$ and numerical 6 -vector $\vec{\Gamma}$ in this kinetic equation can be easily obtained from Eq. (3); see [19].

The solution to the kinetic equation (3) provides the linear transform of the vector $\overrightarrow{\mathcal{P}}$ given by the relation

$$
\begin{aligned}
&\left(\begin{array}{cc}
p_{3}(t) & p_{1}(t)-1 / 2-i\left(p_{2}(t)-1 / 2\right) \\
p_{1}(t)-1 / 2+i\left(p_{2}(t)-1 / 2\right) & 1-p_{3}(t)
\end{array}\right)=\left(\begin{array}{cc}
U_{11}(t) & U_{12}(t) \\
U_{21}(t) & U_{22}(t)
\end{array}\right) \\
& \times\left(\begin{array}{cc}
p_{3}(0) & p_{1}(0)-1 / 2-i\left(p_{2}(0)-1 / 2\right) \\
p_{1}(0)-1 / 2+i\left(p_{2}(0)-1 / 2\right) & 1-p_{3}(0)
\end{array}\right)\left(\begin{array}{cc}
U_{11}^{*}(t) & H_{21}^{*}(t) \\
H_{12}^{*}(t) & H_{22}^{*}(t)
\end{array}\right),
\end{aligned}
$$

with the unitary matrix $U(t)=\exp (-i t H)$. For an arbitrary unitary matrix $U(t)$, the time dependence of probabilities $p_{1}(t), p_{2}(t)$, and $p_{3}(t)$ provides the trajectory of the probability distribution on the simplex, which respects the constraints corresponding to quantum correlations of spin projections on the perpendicular directions given by axes $x$, $y$, and $z$.

The $2 \times 2$ matrix $\rho$ can be mapped onto a column 4 -vector $\vec{\rho}$ with four components $\rho_{1}=\rho_{11}, \rho_{2}=\rho_{12}, \rho_{3}=\rho_{21}$, and $\rho_{4}=\rho_{22}$. In view of the map described, the unitary evolution of the matrix $\rho(t) \rightarrow u(t) \rho(0) u^{\dagger}(t)$ can be presented as the evolution of the vector $\vec{\rho}$; namely, the 4 -vector $\vec{\rho}(t)=u(t) \otimes u^{*}(t) \vec{\rho}(0)$. The $4 \times 4$ matrix $u(t) \otimes u^{*}(t)$ is a unitary 
matrix; it transforms the initial probabilities providing the map of the 4-vector $\overrightarrow{\mathcal{P}}(0)$ with components $p_{3}(0), p_{1}(0)-(1 / 2)-i\left[p_{2}(0)-(1 / 2)\right]$, and $p_{1}(0)-(1 / 2)+i\left[p_{2}(0)-(1 / 2)\right]$, $1 \rightarrow p_{3}(0)$ onto a vector $\overrightarrow{\mathcal{P}}(t)$, namely, $\overrightarrow{\mathcal{P}}(t)=u(t) \otimes u^{*}(t) \overrightarrow{\mathcal{P}}(0)$. Here, vectors $\overrightarrow{\mathcal{P}}(t)$ and $\overrightarrow{\mathcal{P}}(0)$ are columns with vector components expressed in terms of probabilities describing the qubit-state density matrix.

We also can get the evolution of such vectors, which are associated with arbitrary quantum channels. In this case, the evolution of the vector with probability components is given by an analogous expression, where the unitary matrix $u(t) \otimes u^{*}(t)$ is replaced by the $4 \times 4$ matrix $V(t)$ of the form associated with the Sudarshan-Mathews-Rau-Kraus transform [28, 29] $V(t)=\sum_{k}\left(S_{k}(t) \otimes S_{k}^{*}(t)\right)$. Here, $S_{k}$ are arbitrary $2 \times 2$ matrices satisfying the condition $\sum_{k} S_{k}^{\dagger} S_{k}=1_{2}$.

For example, if matrices $S_{k}(t)$ are such that $S_{k}(t)=\sqrt{\lambda}_{k} u_{k}(t)$, where $u_{k}(t)$ are unitary $2 \times 2$ matrices and numbers $\lambda_{k}$ such that $0 \leq \lambda_{k} \leq 1$ satisfy the normalization condition $\sum_{k} \lambda_{l}=1$, the transformation of probability vectors $\overrightarrow{\mathcal{P}}(0) \rightarrow \overrightarrow{\mathcal{P}}(t)$ gives the pseudostochastic map [21] of the 6 -vector with the components $\frac{1}{3}\left(p_{1}(0), 1-p_{1}(0), p_{2}(0), 1-\right.$ $\left.p_{2}(0), p_{3}(0), 1-p_{3}(0)\right)$ corresponding to the initial density matrix of the qubit state $\rho(0)$.

The probability 6 -vector $\overrightarrow{\mathcal{P}}(t)$ with components $\frac{1}{3}\left(p_{1}(t), 1-p_{1}(t), p_{2}(t), 1-p_{2}(t), p_{3}(t), 1-\right.$ $\left.p_{3}(t)\right)$ satisfies the linear kinetic equation determined by the GKSL equation. Thus, we can obtain an arbitrary quantum evolution equation like the von Neumann equation or the Gorini-Kossakowski-Sudarshan-Lindblad (GKSL) equation [30, 31] in the form of a kinetic equation for the probability distribution determining quantum states. This approach can be extended to arbitrary spin states as well as to the description of systems with continuous variables like a quantum parametric oscillator.

In the probability representation, the oscillator states are described by quantum symplectic tomograms [32, 33]. Also these states can be described by optical tomograms, which are the probability distributions satisfying the evolution equation in the form of the kinetic equation [34, 35]. 


\section{General Case of Qudits}

The density $N \times N$ matrix of the qudit state $(N$-level atom, spin- $j$ state with $2 j+1=N$ ) has the matrix elements $\rho_{j k}, j, k=1,2, \ldots N$, for which $\rho_{j k}^{*}=\rho_{k j}, \sum_{k} \rho_{k k}=1$, and $\rho \geq 0$, i.e., eigenvectors of the matrix are nonnegative. It was found [13, 14, 15, 23, 25] that the matrix elements of this matrix are expressed in terms of probabilities $0 \leq p_{1,2,3}^{(j k)} \leq 1$ of dichotomic random variables such that

$$
\begin{aligned}
& \rho_{j k}=p_{1}^{(j k)}-(1 / 2)-i\left(p_{2}^{(j k)}-1 / 2\right), \quad j<k, \\
& \rho_{j j}=p_{3}^{j j}, \quad j=1,2, \ldots, N-1, \\
& \rho_{N N}=1-\sum_{j=1}^{N-1} p_{3}^{j j} .
\end{aligned}
$$

There exist other probability distributions for the density matrix [23, 25], which depend linearly on the probabilities $p_{1,2,3}^{(j k)}$. All properties of the probabilities determining qubit states are valid also for qudit states and for any states with $N=1,2, \ldots, \infty$; for example, for the density matrix $\rho_{j k}$ of harmonic oscillator written in the Fock basis. In this case, we have an infinite number of probability distributions $0 \leq p_{1,2,3}^{(j k)} \leq 1$ of dichotomic random variables determining the oscillator quantum state. The kinetic equation for the probability distribution has the form of a von Neumann equation for the oscillator density matrix in the position representation; it reads

$$
i \frac{\partial \rho\left(x, x^{\prime}, t\right.}{\partial t}=-\frac{1}{2}\left(\frac{\partial^{2}}{\partial x^{2}}-\frac{\partial^{2}}{\partial x^{\prime 2}}\right) \rho\left(x, x^{\prime}, t\right)+\frac{x^{2}-x^{2}}{2} \rho\left(x, x^{\prime}, t\right), \quad \hbar=m=\omega=1 .
$$

The density matrix in the Fock basis $\rho_{j k}=\langle j|\hat{\rho}| k\rangle$ is connected with the matrix in the position representation $\left\langle x|\hat{\rho}| x^{\prime}\right\rangle$ as follows:

$$
\rho\left(x, x^{\prime}\right)=\sum_{k, j=0}^{\infty}\langle x \mid k\rangle\langle k|\hat{\rho}| j\rangle\left\langle j \mid x^{\prime}\right\rangle, \quad k, j=0,1,2, \ldots, \infty
$$

this means that the density matrix $\rho\left(x, x^{\prime}, t\right)$ satisfying Eq. (66) is expressed in terms of probabilities $p_{1,2,3}^{(j k)}$ as

$$
\rho\left(x, x^{\prime}, t\right)=\sum_{k, j=0}^{\infty} \frac{e^{\left(x^{2}+x^{2}\right) / 2}}{\sqrt{\pi}} \frac{H_{k}(x) H_{j}\left(x^{\prime}\right)}{\sqrt{k ! j ! 2^{k+j}}} \rho_{k j}(t)
$$

where the matrix elements

$$
\rho_{k j}(t)=p_{1}^{(k j)}(t)-1 / 2-i\left(p_{2}^{(k, j)}(t)-1 / 2\right), \quad k<j, \quad \rho_{k k}(t)=p_{3}^{(k k)}(t)
$$


are expressed in terms of probabilities $p_{1,2,3}^{(j k)}$ satisfying the kinetic equation corresponding to the used quantum evolution equation either for the unitary or nonunitary evolution.

\section{Conclusions}

The authors of this paper have the privilege to discuss with Prof. V. P. Silin the problem of probability representation of quantum evolution equations, especially in connection with the PhD Thesis of V. N. Chernega [24]. Professor V. P. Silin actively participated in the discussion during V. N. Chernega's defence of his PhD Thesis at the Scientific Council of the Lebedev Physical Institute where Prof. V. P. Silin was a permanent member.

Professor V. P. Silin pointed out that the Wigner representation of the density matrix and the kinetic equation for quasidistributions are used in plasma physics as well as in considering the quantum-liquid properties [5, 6, 7, 8, 9, 10] since it was useful namely due to its applications to the important physical effects. In this connection, Prof. V. P. Silin's advice was to apply the approach based on the probability representation of the quantumstate density matrix for both discrete variables like spin projections and for continuous variables like the particle's position and momenta to clarify the advantages of the new approach. New aspects of this approach that we hope to use are the information-entropic characteristics of the density-matrix elements. These characteristics can be expressed in terms of inequalities for the Shannon entropy [36] like the subadditivity condition existing for different probability distributions. These aspects of solutions of the kinetic equation determining quantum states will be considered in future publications. We are grateful to Prof. V. P. Silin for fruitful discussion and advices, and we dedicate this paper to the memory of Prof. V. P. Silin, a great scientist.

\section{References}

[1] E. Schrödinger, Naturwissenchaften, 14, 664 (1926).

[2] L. D. Landau, Z. Physik, 45, 430 (1927).

[3] J. von Neumann, Mathematische Grundlagen der Quantenmechanik, Springer, Berlin (1932). 
[4] V. P. Silin, Zh. Éksp. Teor. Fiz., 23, 641; 649 (1952).

[5] V. P. Silin, "Study of many-body system spectra by the kinetic equation method," PhD Thesis, Lebedev Physical Institute, Moscow (November 16, 1953) in: Proceedings of the Lebedev Physical Institute [in Russian], Nauka, Moscow (1955), Vol. 6, p. 199 http://proceedings.lebedev.ru/0006-1955/.

[6] A. A. Rukhadze and V. P. Silin, Zh. Éksp. Teor. Fiz., 38, 645 (1960) [Sov. Phys. JETP, 11, 463 (1960)].

[7] V. P. Silin, Zh. Éksp. Teor. Fiz., 38, 977 (1960) [Sov. Phys. JETP, 11, 703 (1960)].

[8] V. P. Silin, Zh. Éksp. Teor. Fiz., 30, 421 (1956) [Sov. Phys. JETP, 3, 305 (1956)].

[9] V. P. Silin, Zh. Éksp. Teor. Fiz., 33, 1227 (1958) [Sov. Phys. JETP, 6, 945 (1958)].

[10] V. P. Silin, Zh. Éksp. Teor. Fiz., 35, 1243 (1959) [Sov. Phys. JETP, 8, 870 (1959)].

[11] Yu. L. Klimontovich and V. P. Silin, Zh. Éksp. Teor. Fiz., 40, 1213 (1961) J. Sov. Phys. JETP, 13, 852 (1961).

[12] V. P. Silin, Phys. Metals Metal Res., 3, 193 (1956).

[13] V. N. Chernega, O. V. Man'ko, and V. I. Man'ko, J. Russ. Laser Res., 38, 141 (2017).

[14] V. N. Chernega, O. V. Man'ko, and V. I. Man'ko, J. Russ. Laser Res., 38, 324 (2017).

[15] V. N. Chernega, O. V. Man'ko, and V. I. Man'ko, J. Russ. Laser Res., 38, 416 (2017).

[16] V. N. Chernega, O. V. Man'ko, and V. I. Man'ko, J. Phys.: Conf. Ser., 1071, 012008 (2018).

[17] V. N. Chernega, O. V. Man'ko, and V. I. Man'ko, J. Russ. Laser Res., 39, 128 (2018).

[18] V. N. Chernega, O. V. Man'ko, and V. I. Man'ko, Eur. Phys. J. D, 73, 10 (2019).

[19] A. Avanesov and V. I. Man'ko, "Probability representation of quantum channels," arXiv:1904.03036.

[20] A. Avanesov and Man'ko, Physica A, 533, 121898 (2019). 
[21] D. Chruściński, V. I. Man’ko, G. Marmo, and F. Ventriglia, Phys. Scr., 90, 115202 (2015).

[22] E. O. Kiktenko, A. O. Malyshev, A. S. Mastiukova, et al., "Probability representation of quantum dynamics using pseudostochastic maps," arXiv:1908.03404 [quant-ph].

[23] M. A. Man'ko, V. I. Man'ko, G. Marmo, et al., "Dichotomic probability representation of quantum states," arXiv:1905.10561.

[24] V. N. Chernega, "Probability representation of quantum physics," PhD Thesis, Lebedev Physical Institute, Moscow (2013).

[25] V. I. Man'ko, G. Marmo, F. Ventriglia, and P. Vitale, J. Phys. A: Math. Gen., 50, 335302 (2017).

[26] V. I. Man'ko and O. V. Man'ko, J. Exp. Theor. Phys., 85, 430 (1997).

[27] V. V. Dodonov and V. I. Man'ko, Phys. Lett. A, 239, 335 (1997).

[28] E. C. G. Sudarshan, P. M. Mathews, and J. Rau, Phys. Rev., 121, 920 (1961).

[29] K. Kraus, States, Effects and Operations, Springer, Berlin (1988).

[30] V. Gorini, A. Kossakowski, and E. C. G. Sudarshan, J. Math. Phys., 17, 821 (1976).

[31] G. Lindblad, Commun. Math. Phys., 48, 119 (1976).

[32] S. Mancini, V. I. Man'ko, and P. Tombesi, Phys. Lett. A, 213, 1 (1996).

[33] O. V. Man'ko and V. I. Man'ko, J. Russ. Laser Res., 18, 407 (1997).

[34] Y. A. Korennoy and V. I. Man'ko, J. Russ. Laser Res., 32, 74 (2011).

[35] G. G. Amosov, Y. A. Korennoy, and V. I. Man'ko, Phys. Rev. A, 85, 052119 (2012).

[36] C. E. Shannon, Bell Syst. Tech. J., 27, 379 (1948). 